Bull. Korean Math. Soc. 51 (2014), No. 3, pp. 681-689

http://dx.doi.org/10.4134/BKMS.2014.51.3.681

\title{
GENERAL DECAY FOR A SEMILINEAR WAVE EQUATION WITH BOUNDARY FRICTIONAL AND MEMORY CONDITIONS
}

\author{
Sun Hye PARK
}

\begin{abstract}
In this paper, we investigate the influence of boundary dissipations on decay property of the solutions for a semilinear wave equation with damping and memory condition on the boundary using the multiplier technique.
\end{abstract}

\section{Introduction}

Our goal in this paper is to study the general decay rates of the solution for a semilinear wave equation with boundary damping and memory conditions of the form:

$$
\begin{aligned}
& u^{\prime \prime}-\Delta u+h(\nabla u)=0 \text { in } \Omega \times(0, \infty), \\
& u=0 \text { on } \Gamma_{1} \times(0, \infty), \\
& \frac{\partial u}{\partial \nu}+\int_{0}^{t} k(t-s, x) u^{\prime}(s) d s+g\left(u^{\prime}\right)=0 \text { on } \Gamma_{0} \times(0, \infty), \\
& u(0)=u_{0}, u^{\prime}(0)=u_{1} \text { in } \Omega,
\end{aligned}
$$

where $\Omega$ is a bounded domain in $\mathbb{R}^{n}(n \geq 1)$ with a sufficiently smooth boundary $\Gamma=\Gamma_{0} \cup \Gamma_{1}$. Here $\Gamma_{0}$ and $\Gamma_{1}$ are closed and disjoint, $\nu$ is the unit outward normal to $\Gamma, u^{\prime}=\frac{\partial u}{\partial t}, \Delta u=\sum_{i=1}^{n} \frac{\partial^{2} u}{\partial x_{i}} . h, k$ and $g$ are given functions.

For the last several decades, the boundary stabilization of classical wave equations has generated much interest and consideration in the literature. Indeed, when $h=0$ and $k(t, x)=0$, the problem has been treated many authors $[8,14,15]$ for linear cases and $[7,9,16]$ for nonlinear ones. Aassila et al. [1] studied problem (1.1)-(1.4), with $h=0$ and a kernel $k$ of exponential decay, and established some decay results. The stability of systems with $h \neq 0$ is

Received January 28, 2013; Revised May 14, 2013.

2010 Mathematics Subject Classification. 35L70, 35B40, 35B37.

Key words and phrases. wave equation, boundary damping, memory condition, general decay rate, Lyapunov functional.

This research was supported by Basic Science Research Program through the National Research Foundation of Korea(NRF) funded by the Ministry of Education, Science and Technology (No. 20110007870). 
somewhat delicate due to the lack of dissipativity (see e.g. [5, 13]). Guesmia [5] investigated the stability to the problem (1.1)-(1.4) with $k=0$ and source term by introducing a special inequality. Most of these works are concerned with the exponential and polynomial decay rates when the kernel function $k$ decays exponentially or the dissipative function $g$ has polynomial growth near the origin. On the other hand, most recently general decay results for various systems have been obtained under generalized conditions on the functions $k$ or $g$. For the related problems, we refer $[2,3,6,9,10,11]$. Messaoudi and Soufyane [12] considered the following wave equation

$$
\left\{\begin{array}{l}
u^{\prime \prime}-\Delta u+f_{1}(u)=0 \text { in } \Omega \times(0, \infty), \\
u=0 \text { on } \Gamma_{1} \times(0, \infty), \\
u=-\int_{0}^{t} f_{2}(t-s) \frac{\partial u}{\partial \nu}(s) d s \text { on } \Gamma_{0} \times(0, \infty), \\
u(0)=u_{0}, u^{\prime}(0)=u_{1} \text { in } \Omega .
\end{array}\right.
$$

By establishing some relations between the relaxation function $f_{2}$ and the corresponding resolvent kernel, they proved a general decay result, which is more general than those usually found in the literature. Motivated by these results, in this work we prove general decay of energy to the problem (1.1)-(1.4) by applying the frameworks of [12] with some necessary modification due to the nature of the problem treated here. It is worth to mention that the results in this paper improve some of those given in $[1,5]$ from some aspects. The remaining part of this paper is organized as follows. In Section 2, we give some notations and material needed for our work and state main results. Section 3 is devoted to investigate the decay of the solution energy.

\section{Statement of main results}

In this section, we present some material needed in the proof of our result and state main result. For a Banach space $X,\|\cdot\|_{X}$ denotes the norm of $X$. Let us consider the Hilbert space $L^{2}(\Omega)$ and $L^{2}\left(\Gamma_{0}\right)$ endowed with the inner products

$$
(u, v)=\int_{\Omega} u(x) v(x) d x, \quad \text { and }(u, v)_{\Gamma_{0}}=\int_{\Gamma_{0}} u(x) v(x) d \Gamma
$$

and the corresponding norm $\|u\|_{L^{2}(\Omega)}^{2}=(u, u)$ and $\|u\|_{L^{2}\left(\Gamma_{0}\right)}^{2}=(u, u)_{\Gamma_{0}}$, respectively. For simplicity, we denote $\|\cdot\|_{L^{2}(\Omega)}$ and $\|\cdot\|_{L^{2}\left(\Gamma_{0}\right)}$ by $\|\cdot\|$ and $\|\cdot\|_{\Gamma_{0}}$, respectively.

We denote

$$
V=\left\{u \in H^{1}(\Omega): u=0 \text { on } \Gamma_{1}\right\} .
$$

Let $x^{0}$ be a fixed point in $\mathbb{R}^{n}, m=x-x^{0}$ and $R=\max \left\{\left|x-x^{0}\right|: x \in \bar{\Omega}\right\}$. Assume that there exists $0<\delta<1$ such that

$$
\Gamma_{0}=\{x \in \Gamma: m \cdot \nu \geq \delta>0\} \text { and } \Gamma_{1}=\{x \in \Gamma: m \cdot \nu \leq 0\} .
$$


Let $\lambda_{1}$ and $\lambda$ be the smallest positive constants such that

$$
\|u\|^{2} \leq \lambda\|\nabla u\|^{2} \text { and }\|u\|_{\Gamma_{0}}^{2} \leq \lambda_{1}\|\nabla u\|^{2}, \quad \forall u \in V
$$

Now, we state the assumptions for the problem (1.1)-(1.4).

$\left(\mathbf{H}_{1}\right)$ Let $h: \mathbb{R}^{n} \rightarrow \mathbb{R}$ be a $C^{1}$ function such that $\nabla h$ is bounded and there exists $\beta>0$ satisfying that

$$
|h(x)| \leq \beta|x| \text { for all } x \in \mathbb{R}^{n} .
$$

$\left(\mathbf{H}_{\mathbf{2}}\right)$ Assume that $g: \mathbb{R} \rightarrow \mathbb{R}$ is a $C^{1}$ function verifying $s g(s)>0$ for $s \neq 0$ and there exist positive constants $\mu_{1}$ and $\mu_{2}$ such that $\mu_{2} \geq \mu_{1}$ and

$$
\mu_{1}|s| \leq|g(s)| \leq \mu_{2}|s| \text { for all } s \in \mathbb{R} .
$$

$\left(\mathbf{H}_{3}\right)$ Let $k \in C^{2}\left(\mathbb{R}_{+} ; L^{\infty}\left(\Gamma_{1}\right)\right)$ such that

$$
k(0, x)>0, \quad k(t, x) \geq 0, \quad k^{\prime}(t, x) \leq 0, \quad k^{\prime \prime}(t, x) \geq-\zeta(t) k^{\prime}(t, x),
$$

where $k^{\prime}(t, x)$ denotes the derivative with respect to $t, \zeta: \mathbb{R}_{+} \rightarrow(0, \infty)$ is a nonincreasing function satisfying $\zeta(t) \geq \zeta_{0}$ for some $\zeta_{0}>0$.

We denote $K(t)=\|k(t)\|_{L^{\infty}\left(\Gamma_{1}\right)}$ for $t \geq 0$.

Examples. (1) Let

$$
k(t, x)=k(t)=\frac{e^{-t}}{(e+t)(\ln (e+t))^{p}}, \text { where } p>0 .
$$

Then simple calculations yield that $k$ satisfies all the conditions in $\left(\mathrm{H}_{3}\right)$ with

$$
\zeta(t)=1+\frac{\ln (e+t)+p}{(e+t) \ln (e+t)} .
$$

(2) $k(t)=\frac{e^{-t}}{(1+t)^{p}}$, where $p>0$, is also an example verifying $\left(\mathrm{H}_{3}\right)$ with

$$
\zeta(t)=1+\frac{p}{1+t}+\frac{p}{(1+t)(1+t+p)} .
$$

According to previous results existing in the literature (see e.g. [4]), we can state the following existence result of the solution subject to (1.1)-(1.4) under the conditions $\left(\mathrm{H}_{1}\right)-\left(\mathrm{H}_{4}\right)$ :

Theorem 2.1. For the initial data $\left(u_{0}, u_{1}\right) \in V \times L^{2}(\Omega)$, the problem (1.1)(1.4) has a unique weak solution $u$ in the class

$$
u \in C(0, T ; V) \cap C^{1}\left(0, T ; L^{2}(\Omega)\right) .
$$

Furthermore, $\left(u_{0}, u_{1}\right) \in\left(V \cap H^{2}(\Omega)\right) \times V$ with the compatibility conditions $\frac{\partial u_{0}}{\partial \nu}+g\left(u_{1}\right)=0$ on $\Gamma_{0}$, the problem (1.1)-(1.4) has a unique solution $u$ in the class

$$
u \in L^{\infty}\left(0, T ; V \cap H^{2}(\Omega)\right), \quad u^{\prime} \in L^{\infty}(0, T ; V), \quad u^{\prime \prime} \in L^{\infty}\left(0, T ; L^{2}(\Omega)\right) .
$$

Our main result is the following. 
Theorem 2.2. If $\beta$ and $K(0)=\|k(0)\|_{L^{\infty}\left(\Gamma_{1}\right)}$ are sufficiently small, there exist $C_{0}>0$ and $\omega>0$ such that

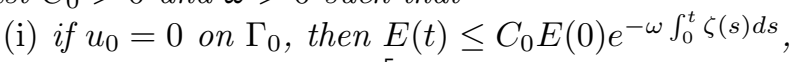

(ii) otherwise, $E(t) \leq C_{0}\left[E(0)+\left\|u_{0}\right\|_{\Gamma_{0}}^{2} \int_{0}^{t} K^{2}(s) e^{\omega \int_{0}^{s} \zeta(\tau) d \tau} d s\right] e^{-\omega \int_{0}^{t} \zeta(s) d s}$, where

$$
E(t)=\frac{1}{2}\left\|u^{\prime}\right\|^{2}+\frac{1}{2}\|\nabla u\|^{2}+\frac{1}{2} \int_{\Gamma_{0}} k(t, x)|u(t, x)|^{2} d \Gamma-\frac{1}{2} \int_{\Gamma_{0}} k^{\prime} \square u d \Gamma .
$$

Remark 2.1. Exponential decay, given in earlier literature (see e.g. $[1,5]$ ), is a special case of Theorem 2.2. Indeed, if $k(t, x)=k(t)=e^{-a t}$ for some $a>0$, $\zeta(t)$ equals to the constant $a>0$ and Theorem 2.2 implies exponential decay estimates.

\section{Asymptotic behavior of solutions}

In this section we shall prove the decay rates in Theorem 2.2. From now on, we shall omit $x$ and $t$ in all functions of $x$ and $t$ if there is no ambiguity. $c$ denotes an arbitrary positive constant independent of $t$ and $x$, which may be different from line to line and even in the same line.

Integration by parts yields

$$
\begin{aligned}
& \int_{0}^{t} k(t-s, x) u^{\prime}(s, x) d s \\
= & k(0, x) u(t, x)-k(t, x) u_{0}(x)+\int_{0}^{t} k^{\prime}(t-s, x) u(s, x) d s \\
= & k(t, x) u(t, x)-k(t, x) u_{0}(x)+\int_{0}^{t} k^{\prime}(t-s, x)(u(s, x)-u(t, x)) d s .
\end{aligned}
$$

Multiplying (1.1) by $u^{\prime}$, which makes sense because $u^{\prime}(t) \in L^{\infty}(0, T ; V)$, we obtain from $(1.2),(1.3)$ and (3.1) the identity

$$
\begin{aligned}
& \frac{1}{2} \frac{d}{d t}\left(\left\|u^{\prime}\right\|^{2}+\|\nabla u\|^{2}\right) \\
= & -\left(h(\nabla u), u^{\prime}\right)-\left(g\left(u^{\prime}\right), u^{\prime}\right)_{\Gamma_{0}}-\left(k(0) u-k(t) u_{0}+\int_{0}^{t} k^{\prime}(t-s) u(s) d s, u^{\prime}\right)_{\Gamma_{0}} .
\end{aligned}
$$

On the other hand, a direct calculation gives

$$
\begin{aligned}
& \int_{0}^{t} k^{\prime}(t-s, x) u(s, x) u^{\prime}(t, x) d s \\
= & -\frac{1}{2} k^{\prime}(t, x)|u(t, x)|^{2}+\frac{1}{2} k^{\prime \prime} \square u \\
& -\frac{1}{2} \frac{d}{d t}\left(k^{\prime} \square u-k(t, x)|u(t, x)|^{2}+k(0, x)|u(t, x)|^{2}\right),
\end{aligned}
$$


where $k^{\prime} \square u=\int_{0}^{t} k^{\prime}(t-s, x)|u(t, x)-u(s, x)|^{2} d s$. Applying this to (3.2), we get

$$
\begin{aligned}
\frac{d}{d t} E(t)= & -\left(h(\nabla u), u^{\prime}\right)-\left(g\left(u^{\prime}\right), u^{\prime}\right)_{\Gamma_{0}}+\int_{\Gamma_{0}} k(t, x) u_{0}(x) u^{\prime}(t, x) d \Gamma \\
& +\frac{1}{2} \int_{\Gamma_{0}} k^{\prime}(t, x)|u(t, x)|^{2} d \Gamma-\frac{1}{2} \int_{\Gamma_{0}} k^{\prime \prime} \square u d \Gamma .
\end{aligned}
$$

Use (2.3), (2.4), (2.5) and Young inequality to obtain

$$
\begin{aligned}
\frac{d}{d t} E(t) \leq & \frac{\beta}{2}\left\|u^{\prime}\right\|^{2}+\frac{\beta}{2}\|\nabla u\|^{2}+\frac{\mu_{1}}{2}\left\|u^{\prime}\right\|_{\Gamma_{0}}^{2}+\frac{1}{2 \mu_{1}} \int_{\Gamma_{0}} k^{2}(t, x)\left|u_{0}(x)\right|^{2} d \Gamma \\
& +\frac{1}{2} \int_{\Gamma_{0}} k^{\prime}(t, x)|u(t, x)|^{2} d \Gamma-\frac{1}{2} \int_{\Gamma_{0}} k^{\prime \prime} \square u d \Gamma .
\end{aligned}
$$

First, let us define the perturbed modified energy by

$$
L(t)=M E(t)+\Psi(t),
$$

where

$$
\Psi(t)=2\left(u^{\prime}, m \cdot \nabla u\right)+(n-1)\left(u^{\prime}, u\right) .
$$

It is easy to check that

$$
L(t) \sim E(t) \text { for appropriately large } M>0 .
$$

Proposition 3.1. For sufficiently small $\beta>0, K(0)>0$ and large $M>0$, there exist positive constants $\alpha_{1}, \alpha_{2}$ and $\alpha_{3}$ verifying

$$
\frac{d}{d t} L(t) \leq-\alpha_{1} E(t)+\alpha_{2} \int_{\Gamma_{0}} k^{2}(t, x)\left|u_{0}(x)\right|^{2} d \Gamma-\alpha_{3} \int_{\Gamma_{0}} k^{\prime} \square u d \Gamma .
$$

Proof. Using (1.1)-(1.4), we have

$$
\begin{aligned}
\Psi^{\prime}(t)= & \int_{\Gamma_{0}}(m \cdot \nu)\left|u^{\prime}\right|^{2} d \Gamma-\left\|u^{\prime}\right\|^{2}+\left(\frac{\partial u}{\partial \nu}, 2 m \cdot \nabla u+(n-1) u\right)_{\Gamma_{0}} \\
& +\left(2 m \cdot \nabla u, \frac{\partial u}{\partial \nu}\right)_{\Gamma_{1}}-\int_{\Gamma}(m \cdot \nu)|\nabla u|^{2} d \Gamma-\|\nabla u\|^{2} \\
& -(2 m \cdot \nabla u+(n-1) u, h(\nabla u)) .
\end{aligned}
$$

Noting that

$$
u=0, \frac{\partial u}{\partial x_{i}}=\nu_{i} \frac{\partial u}{\partial \nu}(i=1, \ldots, n) \text { and } m \cdot \nu \leq 0 \text { on } \Gamma_{1},
$$

we have

$$
\begin{aligned}
\Psi^{\prime}(t) \leq & \int_{\Gamma_{0}}(m \cdot \nu)\left|u^{\prime}\right|^{2} d \Gamma-\left\|u^{\prime}\right\|^{2}+\left(\frac{\partial u}{\partial \nu}, 2 m \cdot \nabla u+(n-1) u\right)_{\Gamma_{0}} \\
& -\int_{\Gamma_{0}}(m \cdot \nu)|\nabla u|^{2} d \Gamma-\|\nabla u\|^{2}-(2 m \cdot \nabla u+(n-1) u, h(\nabla u)) .
\end{aligned}
$$


Young inequality, (2.2) and (2.3) give

$$
\left(\frac{\partial u}{\partial \nu}, 2 m \cdot \nabla u+(n-1) u\right)_{\Gamma_{0}} \leq \eta\|\nabla u\|_{\Gamma_{0}}^{2}+\eta\|\nabla u\|^{2}+C_{\eta}\left\|\frac{\partial u}{\partial \nu}\right\|_{\Gamma_{0}}^{2}
$$

and

$$
(2 m \cdot \nabla u+(n-1) u, h(\nabla u)) \leq \beta(2 R+(n-1) \lambda)\|\nabla u\|^{2},
$$

where $C_{\eta}>0$ is a constant depending on $\eta, \delta, R, n$ and $\lambda_{1}$.

Substituting these into (3.10) and using (2.1), we see that

$$
\begin{aligned}
\Psi^{\prime}(t) \leq & \int_{\Gamma_{0}}(m \cdot \nu)\left|u^{\prime}\right|^{2} d \Gamma-\left\|u^{\prime}\right\|^{2}-(\delta-\eta) \int_{\Gamma_{0}}|\nabla u|^{2} d \Gamma \\
& -(1-\eta-\beta(2 R+(n-1) \lambda))\|\nabla u\|^{2}+C_{\eta}\left\|\frac{\partial u}{\partial \nu}\right\|_{\Gamma_{0}}^{2} .
\end{aligned}
$$

From (3.4) and (3.13), it follows that

$$
\begin{aligned}
\frac{d}{d t} L(t)= & M E^{\prime}(t)+\Psi^{\prime}(t) \\
\leq & \frac{M \beta}{2}\left\|u^{\prime}\right\|^{2}+\frac{M \beta}{2}\|\nabla u\|^{2}-M\left(\mu_{1}-\frac{1}{2}\right)\left\|u^{\prime}\right\|_{\Gamma_{0}}^{2} \\
& +\frac{M}{2} \int_{\Gamma_{0}} k^{2}(t, x)\left|u_{0}(x)\right|^{2} d \Gamma \\
& +\frac{M}{2} \int_{\Gamma_{0}} k^{\prime}(t, x)|u(t, x)|^{2} d \Gamma-\frac{M}{2} \int_{\Gamma_{0}} k^{\prime \prime} \square u d \Gamma \\
& +\int_{\Gamma_{0}}(m \cdot \nu)\left|u^{\prime}\right|^{2} d \Gamma-\left.\left\|u^{\prime}\right\|\right|^{2}-(\delta-\eta) \int_{\Gamma_{0}}|\nabla u|^{2} d \Gamma \\
& -(1-\eta-\beta(2 R+(n-1) \lambda))\|\nabla u\|^{2}+C_{\eta}\left\|\frac{\partial u}{\partial \nu}\right\|_{\Gamma_{0}}^{2} \\
\leq & -\left.\left(1-\frac{M \beta}{2}\right)\left\|u^{\prime}\right\|\right|^{2}-\left(1-\frac{M \beta}{2}-\eta-\beta(2 R+(n-1) \lambda)\right)\|\nabla u\|^{2} \\
& -\left.\frac{\mu_{1} M}{2}\left\|u^{\prime}\right\|\right|_{\Gamma_{0}} ^{2}+\frac{M}{2 \mu_{1}} \int_{\Gamma_{0}} k^{2}(t, x)\left|u_{0}(x)\right|^{2} d \Gamma+\int_{\Gamma_{0}}(m \cdot \nu)\left|u^{\prime}\right|^{2} d \Gamma \\
& -(\delta-\eta) \int_{\Gamma_{0}}|\nabla u|^{2} d \Gamma+\left.C_{\eta}\left\|\frac{\partial u}{\partial \nu}\right\|\right|_{\Gamma_{0}} ^{2} \cdot
\end{aligned}
$$

Eq.(3.1), Cauchy-Swartz inequality and $\left(\mathrm{H}_{3}\right)$ infer that

$$
\begin{gathered}
\left\|\frac{\partial u}{\partial \nu}\right\|_{\Gamma_{0}}^{2}=|| k(t) u(t)-k(t) u_{0}+\int_{0}^{t} k^{\prime}(t-s)(u(s)-u(t)) d s-g\left(u^{\prime}\right)||_{\Gamma_{0}}^{2} \\
\leq c\left[\int_{\Gamma_{0}} k^{2}(t, x)|u(t, x)|^{2} d \Gamma+\int_{\Gamma_{0}} k^{2}(t, x)\left|u_{0}(x)\right|^{2} d \Gamma\right. \\
\left.\quad-\int_{\Gamma_{0}} k(0, x) k^{\prime} \square u d \Gamma+\left\|g\left(u^{\prime}\right)\right\|_{\Gamma_{0}}^{2}\right]
\end{gathered}
$$




$$
\begin{aligned}
\leq c & {\left[K^{2}(0) \int_{\Gamma_{0}}|u(t, x)|^{2} d \Gamma+\int_{\Gamma_{0}} k^{2}(t, x)\left|u_{0}(x)\right|^{2} d \Gamma\right.} \\
& \left.-K(0) \int_{\Gamma_{0}} k^{\prime} \square u d \Gamma+\mu_{2}^{2}\left\|u^{\prime}\right\|_{\Gamma_{0}}^{2}\right] .
\end{aligned}
$$

Combining (3.14) and (3.15), we get

$$
\begin{aligned}
\frac{d}{d t} L(t) \leq & -c_{1}\left\|u^{\prime}\right\|^{2}-c_{2}\|\nabla u\|^{2}-c_{3}\left\|u^{\prime}\right\|_{\Gamma_{0}}^{2}-(\delta-\eta) \int_{\Gamma_{0}}|\nabla u|^{2} d \Gamma \\
& +\left(\frac{M}{2 \mu_{1}}+C_{\eta}\right) \int_{\Gamma_{0}} k^{2}(t, x)\left|u_{0}(x)\right|^{2} d \Gamma-C_{\eta} K(0) \int_{\Gamma_{0}} k^{\prime} \square u d \Gamma,
\end{aligned}
$$

where $c_{1}=1-\frac{M \beta}{2}, c_{2}=1-\frac{M \beta}{2}-\eta-\beta(2 R+(n-1) \lambda)-C_{\eta} K^{2}(0), c_{3}=$ $\frac{\mu_{1} M}{2}-R-\mu_{2}^{2}$.

Taking $\eta$ such that $0<\eta<\delta$ and $M>0$ large enough such that $c_{3}>0$, and then choosing $\beta$ and $K(0)$ sufficiently small so that $c_{1}>0$ and $c_{2}>0$, we complete the proof of Proposition 3.1.

Continuity of the proof of Theorem 2.2. Multiplying (3.8) by $\zeta(t)$ and using (2.5) and (3.4), we obtain

$$
\begin{aligned}
\zeta(t) \frac{d}{d t} L(t) \leq & -\alpha_{1} \zeta(t) E(t)+\alpha_{2} \zeta(t) \int_{\Gamma_{0}} k^{2}(t, x)\left|u_{0}\right|^{2} d \Gamma-\alpha_{3} \zeta(t) \int_{\Gamma_{0}} k^{\prime} \square u d \Gamma \\
\leq & -\alpha_{1} \zeta(t) E(t)+\alpha_{2} \zeta(t) K^{2}(t) \int_{\Gamma_{0}}\left|u_{0}\right|^{2} d \Gamma+\alpha_{3} \int_{\Gamma_{0}} k^{\prime \prime} \square u d \Gamma \\
\leq & -\alpha_{1} \zeta(t) E(t)+\alpha_{2} \zeta(t) K^{2}(t) \int_{\Gamma_{0}}\left|u_{0}\right|^{2} d \Gamma \\
& +\alpha_{3}\left(-2 E^{\prime}(t)-\mu_{1}|| u^{\prime}\left\|_{\Gamma_{0}}^{2}+\beta\right\| u^{\prime}\left\|^{2}+\beta\right\| \nabla u \|^{2}\right. \\
& \left.+\frac{1}{\mu_{1}} \int_{\Gamma_{0}} k^{2}(t, x)\left|u_{0}\right|^{2} d \Gamma\right) .
\end{aligned}
$$

Noting that $\zeta^{\prime}(t) \leq 0$ and $\zeta(t) \geq \zeta_{0}$, it follows that

$$
\begin{aligned}
& \frac{d}{d t}\left(\zeta(t) L(t)+2 \alpha_{3} E(t)\right) \\
\leq & -\alpha_{1} \zeta(t) E(t)+c K^{2}(t) \int_{\Gamma_{0}}\left|u_{0}\right|^{2} d \Gamma+\alpha_{3} \beta\left\|u^{\prime}\right\|^{2}+\alpha_{3} \beta\|\nabla u\|^{2} \\
\leq & -\left(\alpha_{1}-2 \alpha_{3} \beta / \zeta_{0}\right) \zeta(t) E(t)+c K^{2}(t) \int_{\Gamma_{0}}\left|u_{0}\right|^{2} d \Gamma .
\end{aligned}
$$

Define

$$
\mathcal{L}(t)=\zeta(t) L(t)+2 \alpha_{3} E(t)
$$

then it is easy to show that $\mathcal{L}(t)$ is equivalent to $E(t)$ by using (3.7) and the fact $\zeta$ is nonincreasing. Thus, choosing $\beta>0$ sufficiently small again such that 
$\alpha_{1}-2 \alpha_{3} \beta / \zeta_{0}>0$, we arrive at

$$
\mathcal{L}^{\prime}(t) \leq-\alpha \zeta(t) \mathcal{L}(t)+c K^{2}(t)\left\|u_{0}\right\|_{\Gamma_{0}}^{2} \text { for some } \alpha>0
$$

Case (i) : If $u_{0}=0$ on $\Gamma_{0},(3.19)$ becomes

$$
\mathcal{L}^{\prime}(t) \leq-\alpha \zeta(t) \mathcal{L}(t)
$$

Integration this over $(0, t)$ gives

$$
\mathcal{L}(t) \leq \mathcal{L}(0) e^{-\alpha \int_{0}^{t} \zeta(s) d s} .
$$

Case (ii): If $u_{0} \neq 0$ on $\Gamma_{0}$, we put

$$
\mathcal{F}(t):=\mathcal{L}(t)-c\left\|u_{0}\right\|_{\Gamma_{0}}^{2} e^{-\alpha \int_{0}^{t} \zeta(s) d s} \int_{0}^{t} K^{2}(s) e^{\alpha \int_{0}^{s} \zeta(\tau) d \tau} d s .
$$

Then, it holds that

$$
\mathcal{F}^{\prime}(t) \leq-\alpha \zeta(t) \mathcal{F}(t)
$$

and hence

$$
\mathcal{F}(t) \leq \mathcal{F}(0) e^{-\alpha \int_{0}^{t} \zeta(s) d s}
$$

This yields that

$$
\mathcal{L}(t) \leq\left(\mathcal{L}(0)+c\left\|u_{0}\right\|_{\Gamma_{0}}^{2} \int_{0}^{t} K^{2}(s) e^{\alpha \int_{0}^{s} \zeta(\tau) d \tau} d s\right) e^{-\alpha \int_{0}^{t} \zeta(s) d s} .
$$

Consequently, the equivalent relations of $\mathcal{L}, L$, and $E$ and (3.21), (3.25) yield the results in Theorem 2.2 .

\section{References}

[1] M. Aassila, M. M. Cavalcanti, and J. A. Soriano, Asymptotic stability and energy decay rates for solutions of the wave equation with memory in a star-shaped domain, SIAM J. Control Optim. 38 (2000), no. 5, 1581-1602.

[2] F. Alabau-Boussouira, Convexity and weighted integral inequalities for energy decay rates of nonlinear dissipative hyperbolic systems, Appl. Math. Optim. 51 (2005), no. 1, 61-105.

[3] M. M. Cavalcanti, V. N. Domingos Cavalcanti, and P. Martinez, General decay rate estimates for viscoelastic dissipative systems, Nonlinear Anal. 68 (2008), no. 1, 177193.

[4] M. M. Cavalcanti and A. Guesmia, General decay rates of solutions to a nonlinear wave equation with boundary conditions of memory type, Differential Integral Equations 18 (2005), no. 5, 583-600.

[5] A. Guesmia, A new approach of stabilization of nondissipative distributed systems, SIAM J. Control Optim. 42 (2003), no. 1, 24-52.

[6] A. Guesmia and S. A. Messaoudi, General energy decay estimates of Timoshenko systems with frictional versus viscoelastic damping, Math. Methods Appl. Sci. 32 (2009), no. $16,2102-2122$.

[7] V. Komornik, Exact Controllability and Stabilization: The Multiplier Method, John Wiley and Sons, Masson, 1994.

[8] V. Komornik and E. Zuazua, A direct method for the boundary stabilization of the wave equation, J. Math. Pures Appl. (9) 69 (1990), no. 1, 33-54. 
[9] I. Lasiecka and D. Tataru, Uniform boundary stabilization of semilinear wave equations with nonlinear boundary damping, Differential Integral Equations 6 (1993), no. 3, 507533.

[10] P. Martinez, A new method to obtain decay rate estimates for dissipative systems, ESAIM Control Optim. Calc. Var. 4 (1999), 419-444.

[11] S. A. Messaoudi, General decay of solutions of a viscoelastic equation, J. Math. Anal. Appl. 341 (2008), no. 2, 1457-1467.

[12] S. A. Messaoudi and A. Soufyane, General decay of solutions of a wave equation with a boundary control of memory type, Nonlinear Anal. R.W.A. 11 (2010), no. 4, 2896-2904.

[13] J. Y. Park and S. H. Park, On solutions for a hyperbolic system with differential inclusion and memory source term on the boundary, Nonlinear Anal. 57 (2004), no. 3, 459-472.

[14] S. H. Park, J. Y. Park, and J. M. Jeong, Boundary stabilization of hyperbolic hemivariational inequalities, Acta Appl. Math. 104 (2008), no. 2, 139-150.

[15] R. Triggiani, Wave equation on a bounded domain with boundary dissipation: An operator approach, J. Math. Anal. Appl. 137 (1989), no. 2, 438-461.

[16] E. Zuazua, Uniform stabilization of the wave equation by nonlinear boundary feedback, SIAM J. Control Optim. 28 (1990), no. 2, 466-477.

Department of Mathematics

Pusan National University

Busan 609-735, Korea

E-mail address: sh-park@pusan.ac.kr 